\title{
Principal Bundle Maps via Rational Homotopy Theory
}

By

Hiroo ShigA* and Toshihiro Yamaguchi**

\begin{abstract}
Let $P$ be a finite complex on which $S^{1}$ acts freely. In this paper, we shall give a sufficient condition that the kernel of the natural map (forgetful map) Aut $_{S^{1}} P \rightarrow$ Aut $P$ is a finite group.
\end{abstract}

\section{$\S 1 . \quad$ Introduction}

Let

$$
S^{1} \rightarrow P \stackrel{\pi}{\rightarrow} B
$$

be a principal $S^{1}$-bundle over the base space $B$. We denote by autP (resp. $a_{S^{1}} P$ ) the space of self homotoy equivalences of $P$ (resp. the space of $S^{1}$ equivariant self homotopy equivalences of $P$ ). Let Aut $P\left(\right.$ resp. $\operatorname{Aut}_{S^{1}} P$ ) be the group of path connected components of autP $\left(\right.$ resp. aut $\left.{ }_{S^{1}} P\right)$. Then we have a natural homomorphism

$$
\mathcal{F}: \operatorname{Aut}_{S^{1}} P \rightarrow \operatorname{Aut} P
$$

obtained by forgetting $S^{1}$-action, which is called forgetful map. The kernel Ker $\mathcal{F}$ was discussed in [5, Problem 13] and [9]. There are the examples where Ker $\mathcal{F}$ are not zero but finite, countable and uncountable [8]. In this paper we assume that $B$ is a connected, simply connected and finite complex. We study $\mathcal{F}$ from the view point of rational homotopy theory. We prove

Communicated by K. Saito. Received November 8, 2001.

2000 Mathematics Subject Classification(s): 55P10, 55P62, 55R10.

*Department of Mathematical Science, College of Science, Ryukyu University, Nishiharachou, Okinawa 903-0213, Japan.

** Mathematics Education, Faculty of Education, Kochi University, Akebono-cho, Kochi 780-8520, Japan. 
Theorem 1.1. $\quad$ Suppose $\pi_{2}\left(\right.$ aut $\left._{I d} B\right) \otimes Q=0$. Then $\operatorname{Ker} \mathcal{F}$ is a finite group for any principal $S^{1}$-bundle over $B$, where aut ${ }_{I d} B$ is the identity component of autB.

In particular, by [7] we have

Corollary 1.2. If the base $B$ has the rational homotopy type of the homogeneous space $G / U$ with $\operatorname{rank} G=\operatorname{rank} U$, then $\operatorname{Ker} \mathcal{F}$ is a finite group for any principal $S^{1}$-bundle over $B$.

The outline of the proof of Theorem 1.1 goes as follows:

We recall [Gottlieb] that the group $\operatorname{Aut}_{S^{1}} P$ is isomorphic to the subgroup Aut $_{k} B$ of Aut $B$, which preserves the homotopy class of the classifying map of $P, k: B \rightarrow B S^{1}$.

In rational homotopy theory, Aut $P$ and $\operatorname{Aut}_{k} B$ correspond to $\operatorname{Aut}_{Q} \mathcal{M}(P)$ and $\operatorname{Aut}_{Q}^{t} \mathcal{M}(B)$ respectively, where $\mathcal{M}(X)$ is the minimal model of a space $X$ and $\operatorname{Aut}_{Q} \mathcal{M}(X)$ denotes the group of D.G.A. homotopy classes of automorphisms of $\mathcal{M}(X)$. (See the detail in Section 2.)

Then we shall show that a certain homomorphism $\left[\pi_{t}\right]: \operatorname{Aut}_{Q}^{t} \mathcal{M}(B) \rightarrow$ $\operatorname{Aut}_{Q} \mathcal{M}(P)$ corresponds to $\mathcal{F}$ (see Proposition 2.2) and we show that $\operatorname{Ker}\left[\pi_{t}\right]=$ $I d_{\mathcal{M}(B)}$ is zero if the 0 -dimensional homology of certain derivations $H_{0}\left(\right.$ Der $^{t}$ $(\mathcal{M}(B),(t)))$ is trivial (see Proposition 2.3). We then prove that $H_{0}\left(D_{e r}{ }^{t}\right.$ $(\mathcal{M}(B),(t)))=0$ if $\pi_{2}\left(\right.$ aut $\left._{I d} B\right) \otimes Q=0$. Finally by Sullivan's theorem ([6, p. 307 Theorem $10.2(\mathrm{i})])$ if $\operatorname{Ker}\left[\pi_{t}\right]=I d_{\mathcal{M}(B)}$ then $\operatorname{Ker} \mathcal{F}$ is finite.

This paper is organized as follows: In Section 2 we study $\mathcal{F}$ by using automorphism group of minimal models when the bundle (1.1) is not trivial (Proposition 2.2) and then prove Proposition 2.3. In Section 3 we prove Theorem 1.1 and give some examples.

\section{$\S 2 . \quad B u n d l e$ Maps and Automorphism Groups of Minimal Models}

Let $k: B \rightarrow B S^{1}$ be the classifying map of (1.1). There is a Serre fibration $([1]):$

$$
\operatorname{map}\left(B, S^{1}\right) \rightarrow \operatorname{aut}_{S^{1}} P \stackrel{\psi}{\rightarrow} \operatorname{aut}_{k} B,
$$

where $\operatorname{map}\left(B, S^{1}\right)$ denotes the space of maps $B \rightarrow S^{1}$ and $\operatorname{aut}_{k} B$ is the subspace of aut $B$ consisting of all $f \in$ aut $B$ satisfying the condition $k \circ f \simeq k$. Since $B$ is simply conected, $\operatorname{map}\left(B, S^{1}\right)$ is connected and $\psi$ induces the isomorphism

$$
\pi_{0}(\psi): \operatorname{Aut}_{S^{1}} P \cong \operatorname{Aut}_{k} B
$$


where $\operatorname{Aut}_{k} B$ denotes the group of path components of $\operatorname{aut}_{k} B$. For $f \in a u t_{k} B$ there is an element $\bar{f} \in$ aut $_{S^{1}} P$ such that $\psi(\bar{f})=f$. The diagram

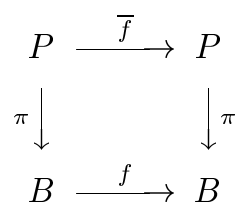

is commutative. We define $T: \operatorname{Aut}_{k} B \rightarrow \operatorname{Aut} P$ by $T([f])=[\bar{f}]$, which is well defined and

$$
\mathcal{F}=T \circ \pi_{0}(\psi) \text {. }
$$

We study $T$ by automorphism groups of Sullivan minimal models ([6]). From now on, we assume that the bundle (1.1) is not trivial. Let $\mathcal{M}(P)=$ $(\wedge V, d)$ be the minimal model of $P$.

Since there is a fibration $P \rightarrow B \rightarrow B S^{1}$, it follows from the non-triviality of (1.1) that the minimal model of $B$ has the form ([3]; p. 206, Theorem 4.6)

$$
\mathcal{M}(B)=(Q[t] \otimes \wedge V, D)
$$

which satisfies the following conditions:

(i) $\operatorname{deg} t=2, D(t)=0$.

(ii) $(Q[t], 0)$ is the minimal model of $B S^{1}$ such that

$$
D(1 \otimes v)=1 \otimes d v+D_{t} v . \quad(*)
$$

(iii) $D_{t} v$ is a decomposable element contained in the ideal $(t \otimes 1)$.

Let $\pi^{*}: \mathcal{M}(B) \rightarrow \mathcal{M}(P)$ be the D.G.A. (differential graded algebra) map induced by the projection $\pi$. Then

$$
\begin{aligned}
& \pi^{*}(1 \otimes v)=v \quad \text { for } v \in \wedge V \\
& \pi^{*}(t \otimes 1)=0 .
\end{aligned}
$$

Let $\operatorname{Aut} \mathcal{M}(B)$ be the group of D.G.A. automorphisms of $\mathcal{M}(B)$ and $\operatorname{Aut}^{t} \mathcal{M}(B)$ be the subgroup of $\operatorname{Aut} \mathcal{M}(B)$ which fixes the element $t \otimes 1$.

Now we quote some results on nilpotent derivations and unipotent autmorphism group ([6]). Let $\left(\mathcal{M}(Y), d_{Y}\right)$ be a minimal model and $\operatorname{Der}_{i} \mathcal{M}(Y)$ be the set of Q-derivations of $\mathcal{M}(Y)$ decreasing the degree by $i$. The boundary operator

$$
\delta_{Y}: \operatorname{Der}_{i} \mathcal{M}(Y) \rightarrow \operatorname{Der}_{i-1} \mathcal{M}(Y)
$$


is defined by

$$
\delta_{Y} \phi=\phi \circ d_{Y}+(-1)^{i+1} d_{Y} \circ \phi, \quad \phi \in \operatorname{Der}_{i} \mathcal{M}(Y) .
$$

Then $\delta_{Y}^{2}=0$.

If $i>0$, any element $\phi$ of $\operatorname{Der}_{i} \mathcal{M}(Y)$ is nilpotent. Hence, for each element $v$ of $\mathcal{M}(Y)$, there is a positive integer $m$ such that $\phi^{m}(v)=0$. If an element $\phi$ of $\operatorname{Der}_{0} \mathcal{M}(Y)$ is nilpotent, $\exp \phi=i d+\phi+\phi^{2} / 2+\cdots+\phi^{n} / n !+\cdots$ is well defined. An element $f \in \operatorname{Aut} \mathcal{M}(Y)$ is D.G.A. homotopic to identity if and only if $f$ can be written as

$$
f=\exp \delta_{Y} \phi
$$

where $\phi$ is a nilpotent derivation of degree one [6, Propositions 6.3 and 6.5]. Then

$$
\operatorname{Aut}_{Q} \mathcal{M}(Y)=\operatorname{Aut} \mathcal{M}(Y) / \exp \left(\delta_{Y} \operatorname{Der}_{1} \mathcal{M}(Y)\right)
$$

represents the group of D.G.A. homotopy classes of D.G.A. autmorphisms.

For an element $f \in \operatorname{Aut}^{t} \mathcal{M}(B)$, we have a D.G.A. endomorphism $\pi_{t}(f)$ of $\mathcal{M}(P)$ defined by

$$
\pi_{t}(f)(v)=\pi^{*}(f(1 \otimes v))
$$

such that the following diagram is commutative:

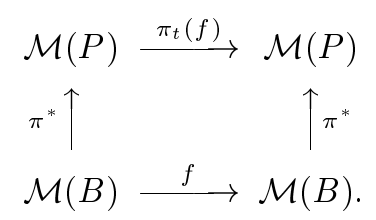

If $g: \mathcal{M}(P) \rightarrow \mathcal{M}(P)$ is a D.G.A. map satisfying the condition $\pi^{*} \circ f=g \circ \pi^{*}$, $g=\pi_{t}(f)$. Hence $\pi_{t}(f) \circ \pi_{t}(g)=\pi_{t}(f \circ g)$ and $\pi_{t}\left(I d_{\mathcal{M}(B)}\right)=I d_{\mathcal{M}(P)}$. Thus $\pi_{t}(f) \in \operatorname{Aut} \mathcal{M}(P)$.

Lemma 2.1. If $f, g \in \operatorname{Aut}^{t} \mathcal{M}(B)$ are D.G.A. homotopic, then so are $\pi_{t}(f)$ and $\pi_{t}(g)$.

Proof. Since $g^{-1} \circ f$ is D.G.A. homotopic to the identity, there exists $\phi \in \operatorname{Der}_{1} \mathcal{M}(B)$ such that $g^{-1} \circ f=\exp \delta_{B} \phi$. Then $\pi_{t}(g)^{-1} \circ \pi_{t}(f)(v)=$ $\pi_{t}\left(g^{-1} \circ f\right)(v)=\pi^{*}\left(\exp \delta_{B} \phi(1 \otimes v)\right)=\exp \left(\delta_{P} \bar{\phi}(v)\right)$ where $\bar{\phi}$, the derivation of $\mathcal{M}(P)$, is defined by $\bar{\phi}(v)=\pi^{*}(\phi(1 \otimes v))$ for $v \in \mathcal{M}(P)$.

We denote by $\operatorname{Aut}_{Q}^{t} \mathcal{M}(X)$ the group of D.G.A. homotopy classes of Aut ${ }^{t}$ $\mathcal{M}(X)$. Then we have 
Proposition 2.2. The following diagram is commutative.

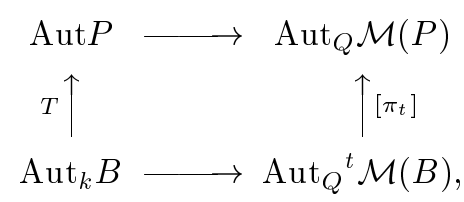

where horizontal maps correspond to the induced maps on the minimal models and $\left[\pi_{t}\right]$ is the induced map from $\pi_{t}$ on D.G.A. homotopy classes.

Proof. Let $f \in$ aut $_{k} B$ and $f^{*} \in \operatorname{Aut}^{t} \mathcal{M}(B)$ the induced map on minimal model. Then $\pi_{t}\left(f^{*}\right)(v)=\pi^{*} f^{*}(1 \otimes v)=T(f)^{*}(v)$ by (2.1). Taking homotopy class, we have the assertion.

Let $f \in \operatorname{Aut}^{t} \mathcal{M}(B)$ be an element such that $[f] \in \operatorname{Ker}\left[\pi_{t}\right]$. Then $\pi_{t}(f)$ is D.G.A. homotopic to identity and it follows from [6, Proposition 6.5] that we may write

$$
\pi_{t}(f)=\exp \delta_{P} \psi
$$

for some $\psi \in \operatorname{Der}_{1} \mathcal{M}(P)$. Define $\tilde{\psi} \in \operatorname{Der}_{1} \mathcal{M}(B)$ by

$$
\begin{aligned}
\tilde{\psi}(1 \otimes v) & =1 \otimes \psi(v) \quad \text { for } v \in \wedge V \\
\tilde{\psi}(t \otimes 1) & =0 .
\end{aligned}
$$

Now consider the element

$$
\tilde{f}=\left(\exp \delta_{B} \tilde{\psi}\right)^{-1} \circ f \in \operatorname{Aut}^{t} \mathcal{M}(B) \quad(* *),
$$

where $\delta_{B} \tilde{\psi}=\tilde{\psi} D+D \tilde{\psi}$. Since $D(1 \otimes v)-1 \otimes d v$ is contained in the ideal $(t \otimes 1)=(t)$ by $(*), \delta_{B} \tilde{\psi}(1 \otimes v)-1 \otimes \delta_{P} \psi(v)$ is also contained in the ideal $(t)$. Then we have:

$$
\begin{aligned}
\tilde{f}(1 \otimes v) & =\left(\exp \delta_{B} \tilde{\psi}\right)^{-1} \circ f(1 \otimes v) \\
& =\left(\exp \delta_{B} \tilde{\psi}\right)^{-1}\left(1 \otimes \pi_{t}(f)(v)+\sum_{j \geq 1} t^{j} \otimes w_{j}\right) \quad\left(w_{j} \in \wedge V\right) \\
& =\left(\exp \delta_{B} \tilde{\psi}\right)^{-1}\left(1 \otimes \exp \delta_{P} \psi(v)+\sum_{j \geq 1} t^{j} \otimes w_{j}\right) \\
& =1 \otimes\left(\exp \delta_{p} \psi\right)^{-1} \circ \exp \delta_{p} \psi(v)+\sum_{j \geq 1} t^{j} \otimes\left(\exp \delta_{P} \psi\right)^{-1}\left(w_{j}\right) \\
& =1 \otimes v+\sum_{j \geq 1} t^{j} \otimes\left(\exp \delta_{P} \psi\right)^{-1}\left(w_{j}\right) .
\end{aligned}
$$


So we take

$$
\tilde{f}-I d_{\mathcal{M}(B)}=X
$$

and

$$
\sigma=\log \left(I d_{\mathcal{M}(B)}+X\right)=X-X^{2} / 2+\cdots .
$$

Then $\sigma \in \operatorname{Der}_{0}^{t}(\mathcal{M}(B),(t))$ and $\delta_{B} \sigma=0$ since $X$ commutes with $D$. Then we can write $\tilde{f}=\exp \sigma$, and by $(* *) f$ and $\tilde{f}$ are D.G.A. homotopic. Here $\operatorname{Der}_{0}^{t}(\mathcal{M}(B),(t))$ denotes the set of degree zero $Q[t]$-derivations of $\mathcal{M}(B)$ with value in the ideal $(t)$. Note that $\operatorname{Der}_{0}^{t}(\mathcal{M}(B),(t))$ forms a Lie algebra by $[\sigma, \tau]=$ $\sigma \circ \tau-\tau \circ \sigma$ for $\sigma, \tau \in \operatorname{Der}_{0}^{t}(\mathcal{M}(B),(t))$ and that any element of it is nilpotent.

Conversely, if we can write $f=\exp \tau$ for $\tau \in \operatorname{Der}_{0}^{t}(\mathcal{M}(B),(t))$ with $\delta_{B} \tau=0$, then $\pi_{t}(f)(v)=\pi^{*}(\exp \tau(1 \otimes v))=v$.

Thus we see that

$$
[\exp ]: Z_{0}(\mathcal{M}(B),(t)) \rightarrow \operatorname{Ker}\left[\pi_{t}\right]
$$

is surjective map, where [exp] is the D.G.A. homotopy class of the composition of the exponential map and $Z_{0}(\mathcal{M}(B),(t))=\left\{\operatorname{Der}_{0}^{t}(\mathcal{M}(B),(t)) ; \delta_{B} \phi=0\right\}$.

Proposition 2.3. If $H_{0}\left(\operatorname{Der}^{t}(\mathcal{M}(B),(t))\right)=0$, then $\operatorname{Ker}\left[\pi_{t}\right]=$ $\left\{\operatorname{Id}_{\mathcal{M}(B)}\right\}$, where $H_{0}\left(\operatorname{Der}^{t}(\mathcal{M}(B),(t))\right)=Z_{0}(\mathcal{M}(B),(t)) / \delta_{B} \operatorname{Der}_{1} \mathcal{M}(B)$ $\cap Z_{0}(\mathcal{M}(B),(t))$.

Proof. We take $\bar{H}_{0}=Z_{0}(\mathcal{M}(B),(t)) / \sim$, where $\sim$ is defined as follows: For $\sigma, \tau \in Z_{0}(\mathcal{M}(B),(t)), \sigma \sim \tau$ if $\exp \sigma$ is D.G.A. homotopic to exp $\tau$, that is, $\exp \sigma \circ \exp (-\tau) \sim I d_{\mathcal{M}(B)}$. By the Baker-Campbell-Hausdorff formula, it is equivalent to

$$
\sigma-\tau+\frac{1}{2}[\sigma, \tau]-\frac{1}{12}[\sigma,[\sigma, \tau]]+\cdots \in \delta_{B} \operatorname{Der}_{1}(\mathcal{M}(B)) .
$$

Let $p: Z_{0}(\mathcal{M}(B),(t)) \rightarrow \bar{H}_{0}$ be the natural map. If $\sigma-\tau=\delta_{B} \psi$ for some $\psi \in \operatorname{Der}_{1}(\mathcal{M}(B))$, we have

$$
[\sigma, \tau]=\left[\sigma, \sigma-\delta_{B} \psi\right]=-\left[\sigma, \delta_{B} \psi\right]=-\delta_{B}[\sigma, \psi] .
$$

Similarly we see that each term of the Baker-Campbell-Hausdorff formula is $\delta_{B^{-}}$ exact. Hence $\sigma \sim \tau$. Thus $p$ induces a surjective map $\bar{p}: H_{0}\left(\operatorname{Der}^{t}(\mathcal{M}(B),(t))\right)$ $\rightarrow \bar{H}_{0}$. If $H_{0}\left(\operatorname{Der}^{t}(\mathcal{M}(B),(t))\right)=0$, then the set $\bar{H}_{0}$ consists of one element (represented by $\delta_{B}$-exact element). Since [exp] induces the bijective correspondence $\bar{H}_{0} \rightarrow \operatorname{Ker}\left[\pi_{t}\right]$, we have $\operatorname{Ker}\left[\pi_{t}\right]=\left\{I d_{\mathcal{M}(B)}\right\}$. 


\section{$\S 3 . \quad$ The Proof of Theorem 1.1 and Examples}

Proposition 3.1. If $H_{2}(\operatorname{Der} \mathcal{M}(B))=0$, then

$$
\left[\pi_{t}\right]: \operatorname{Aut}_{Q}^{t} \mathcal{M}(B) \rightarrow \operatorname{Aut}_{Q} \mathcal{M}(P)
$$

is monomorphic.

Proof. Consider the homomorphism

$$
t_{*}: H_{2}\left(\operatorname{Der}^{t} \mathcal{M}(B)\right) \rightarrow H_{0}\left(\operatorname{Der}^{t}(\mathcal{M}(B),(t))\right)
$$

induced from the multiplication by $t$ with its value. Clearly it is epimorphic. Since

$$
H_{2}\left(\operatorname{Der}^{t} \mathcal{M}(B)\right) \subset H_{2}(\operatorname{Der} \mathcal{M}(B)),
$$

$H_{0}\left(\operatorname{Der}^{t}(\mathcal{M}(B),(t))\right)=0$. Then by Proposition 2.3, $[f]=I d_{\mathcal{M}(B)}$.

Proof of Theorem 1.1. It follows from ([6, p. 313-314]) that $H_{2}(\operatorname{Der} \mathcal{M}$ $(B)) \cong \pi_{2}\left(\operatorname{aut}_{I d}(B)\right) \otimes Q=0$. Hence $\left[\pi_{t}\right]$ is monomorphic by Proposition 3.1. By [6, Theorem 10.2], the kernel of the horizontal maps of the diagram of Proposition 2.2 is finite group. Hence the assertion easily follows when the bundle (1.1) is non-trivial.

Next we consider the case the bundle (1.1) is trivial. Note that (2.2) is not minimal and $\pi_{t}(f)$ is not well-defined in (2.3) in this case. Then $P \simeq S^{1} \times B$ and $\operatorname{Aut}_{S^{1}}\left(S^{1} \times B\right) \cong$ Aut $B$. Then $T$ is monomorphic.

Remark. If $P$ is 2-connected, then $\mathcal{M}^{2}(B)$ is the vector space spanned by $t$. Then

$$
H_{0}\left(\operatorname{Der}^{t}(\mathcal{M}(B),(t))\right) \subset H_{0}\left(\operatorname{Der}\left(\mathcal{M}(B), \mathcal{M}^{+}(B) \cdot \mathcal{M}^{+}(B)\right)\right),
$$

where $\operatorname{Der}\left(\mathcal{M}(B), \mathcal{M}^{+}(B) \cdot \mathcal{M}^{+}(B)\right)$ is the Lie algebra of $Q$-derivations of $\mathcal{M}(B)$ whose values are decomposable elements. This implies that any element of $\operatorname{Ker}\left[\pi_{t}\right]$ induces identity on the rational homotopy group. In particular, if $\operatorname{Aut}_{\sharp} \mathcal{M}(B)=\left\{I d_{\mathcal{M}(B)}\right\}$, Ker $\mathcal{F}$ is a finite group. Here $\operatorname{Aut}_{\sharp} \mathcal{M}(B)$ denotes the group of D.G.A. homotopy classes of D.G.A. automorphisms of $\mathcal{M}(B)$ which induce identity on the rational homotopy group.

In the following three examples the bundles are not trivial, so we can use the result due to S. Halperin [4, Proposition 4.2]: 
Proposition $3.2([4])$. If the minimal models of $P$ and $B$ are given as in (2.2) and $\operatorname{dim}_{Q} H^{*}(\mathcal{M}(B))<\infty$, then $P$ has the same rational homotopy type as a total space of a principal $S^{1}$-bundle over $B$.

Example 3.1. Let

$$
\mathcal{M}(P)=\left(\wedge\left(x_{3}, y_{3}, z_{5}\right), d\right)
$$

with $d(z)=x y, d(x)=d(y)=0, \operatorname{deg} *_{i}=i$ and

$$
\mathcal{M}(B)=\left(\wedge\left(t, x_{3}, y_{3}, z_{5}\right), D\right)
$$

with $D(t)=0, D(x)=D(y)=0, D(z)=x y+t^{3}$. Then $\operatorname{dim} H^{*}(B ; Q)<$ $\infty$ and we can have $\pi_{2}\left(\right.$ aut $\left._{I d} B\right) \otimes Q \cong H_{2}(\operatorname{Der} \mathcal{M}(B))=0$ by straigtfoward calculations.

Example 3.2. $\quad$ In Theorem 1.1, the condition $\pi_{2}\left(\right.$ aut $\left._{I d} B\right) \otimes Q=0$ is not necessary for Ker $\mathcal{F}$ being finite. In fact, let

$$
\mathcal{M}(P)=\left(\wedge\left(x_{3}, y_{3}, z_{5}, w_{9}\right), d\right)
$$

with $d(z)=x y$ and $d(x)=d(y)=d(w)=0$ and

$$
\mathcal{M}(B)=\left(\wedge\left(t, x_{3}, y_{3}, z_{5}, w_{9}\right), D\right)
$$

with $D(z)=x y+t^{3}, D(t)=D(x)=D(y)=D(w)=0$. Then $\operatorname{dim} H^{*}(B ; Q)<$ $\infty$ and $H_{2}(\operatorname{Der} \mathcal{M}(B))$ is the two dimensional vector space spanned by $\left(w, x t^{2}\right)$ and $\left(w, y t^{2}\right)$, where $(u, v)$ denotes the Q-derivation which sends $u$ to $v$ and the other generators to zero. On the other hand, we have $H_{0}\left(\operatorname{Der}^{t}(\mathcal{M}(B),(t))\right)=0$. Hence by Proposition 2.3 Ker $\mathcal{F}$ is finite for $P$.

Example 3.3. There are two principal $S^{1}$-bundles $P_{1}, P_{2}$ over the same base $B$ such that $\operatorname{Ker} \mathcal{F}_{P_{1}}$ is not finite but Ker $\mathcal{F}_{P_{2}}$ is finite. Let

$$
\mathcal{M}\left(P_{1}\right)=\left(\wedge\left(s_{2}, x_{3}, v_{3}, u_{3}, z_{7}, w_{7}\right), d_{1}\right)
$$

with $d_{1}(s)=d_{1}(x)=d_{1}(v)=d_{1}(z)=d_{1}(w)=0, d_{1}(u)=s^{2}$ and

$$
\mathcal{M}\left(P_{2}\right)=\left(\wedge\left(t_{2}, x_{3}, v_{3}, u_{3}, z_{7}, w_{7}\right), d_{2}\right)
$$

with $d_{2}(t)=d_{2}(x)=d_{2}(v)=d_{2}(u)=d_{2}(w)=0, d_{2}(z)=t^{4}$. Let

$$
\mathcal{M}(B)=\left(Q[t] \otimes \mathcal{M}\left(P_{1}\right), D\right)=\left(Q[s] \otimes \mathcal{M}\left(P_{2}\right), D\right)
$$

with $D(s)=D(t)=D(x)=D(w)=0$ and $D(z)=t^{4}, D(v)=s t, D(u)=s^{2}$. Then $\operatorname{dim} H^{*}(B ; Q)<\infty$ and $H_{0}\left(\operatorname{Der}^{t}(\mathcal{M}(B),(t))\right)$ is one dimensional vector space spanned by $\left(w, t^{2} x\right)$. But $H_{0}\left(\operatorname{Der}^{s}(\mathcal{M}(B),(s))\right)=0$. 
Finally we note that the forgetful map can be defined via fiber homotopy equivalences.

Let $P$ be a space on which $S^{1}$ acts (not necessarily free). We consider the fibration

$$
P \rightarrow E S^{1} \times_{S^{1}} P \rightarrow B S^{1},
$$

where $S^{1}$ acts on $E S^{1} \times P$ by the usual manner. Let $\mathcal{L}\left(E S^{1} \times{ }_{S^{1}} P\right)$ be the group of homotopy classes of fiber homotopy equivalence of $E S^{1} \times{ }_{S^{1}} P$. For each $[f] \in \operatorname{Aut}_{S^{1}} P$, we denote $\left[f_{1}\right] \in \mathcal{L}\left(E S^{1} \times_{S^{1}} P\right)$ be the induced map from $i d \times f: E S^{1} \times P \rightarrow E S^{1} \times P$. Hence we have a homomorphism $\psi:$ Aut $_{S^{1}} P \rightarrow$ $\mathcal{L}\left(E S^{1} \times{ }_{S^{1}} P\right)$ by $\psi([f])=\left[f_{1}\right]$. If we recall the natural map $([2]) R: \mathcal{L}\left(E S^{1} \times_{S^{1}}\right.$ $P) \rightarrow$ Aut $P$, then we have

$$
\mathcal{F}=R \circ \psi
$$

\section{References}

[1] Gottlieb, D. H., Applications of bundle map theory, Trans. Amer. Math. Soc., 171 (1972), 23-50.

[2] Félix, Y. and Thomas, J. C., Nilpotent subgroups of the group of fibre homotopy equivalences, Publ. Mat., 39 (1995), 95-106.

[3] Halperin, S., Rational fibrations, minimal models and fibrings of homogeneous spaces, Trans. A.M.S., 244 (1978), 199-223.

[4] - Rational homotopy and torus actions, London Math. Soc. L.N.S., 93 (1985), 293-306.

[5] Kahn, D. W., Some research problems on homotopy-self-equivalences, Springer L.M.N., 1425 (1990), 204-207.

[6] Sullivan, D., Infinitesimal computations in topology, I.H.E.S., 47 (1978), 269-331.

[7] Shiga, H. and Tezuka, M., Rational fibration, homogeneous spaces with positive Euler characteristic and Jacobians, Ann. Inst. Fourier, 37 (1987), 81-106.

[8] Shiga, H., Tsukiyama, K. and Yamaguchi, T., Principal $S^{1}$-bundles and forgetful maps, Contemp. Math., 274 (2001), 293-297.

[9] Tsukiyama, K., Equivariant homotopy equivalences and a forgetful map, Bull. Korean. Math. Soc., 36 (1999), 649-654. 